\title{
Effects of a Depletion Layer on Flow in a Rotating Channel
}

\author{
Chiu-On $\mathrm{Ng}^{1}$ \\ Department of Mechanical Engineering, The University of Hong Kong, Pokfulam Road, Hong Kong
}

\begin{abstract}
An analytical model is presented for pressure-driven two-fluid flow between two parallel flat plates rotating about an axis perpendicular to the plates. The aim is to determine the lubricating effects due to a near-wall low-viscosity depletion layer on the axial as well as transverse flows in a rotating channel. It is found that the primary and secondary flow fields may be affected differently by the system rotation, the thickness of the depletion layer, and the viscosity ratio. For a very thin depletion layer, the wall lubrication reduces to a Navier slip condition, where the slip length is found to be the same as the one for a non-rotating channel at the leading order, while the higher-order slip length is a function of a complex rotation parameter. The Fåhræus-Lindqvist effect in a rotating environment is also examined.
\end{abstract}

Keywords: Rotating channel, depletion layer, slip, Fåhræus-Lindqvist effect

\section{Introduction}

In suspensions or polymer solutions, there usually exists a particle-depleted layer near a wall if the wall is non-adsorbing [1]. The so-called depletion layer owes its presence to the finite size of the suspended particles, which causes the center of these discrete entities in the solution to be excluded from a region next to a non-adsorbing wall. Without the inclusion, the fluid in the depletion layer, essentially the solvent, is significantly less viscous than the bulk fluid, and hence acts as a lubricant to the flow. A good example is blood flow in the microvasculature. The presence of a cell-free layer near the vessel wall contributes to the lowering of effective viscosity in the blood flow through a microvessel, a phenomenon known as the Fåhræus-Lindqvist effect [2].

The notion of a depletion layer is also used to account for dewetting phenomena leading to slip on a hydrophobic surface [3]. In modeling flow over a hydrophobic surface, it is often postulated that the bulk liquid is separated from the surface by a gas layer (e.g., see Vinogradova [4]; de Gennes [5]; Busse et al. [6]). Given the high ratio of viscosity between liquid and gas, even a very thin gas layer

\footnotetext{
${ }^{1}$ E-mail address: cong@hku.hk
}

Preprint submitted to Mechanics Research Communications may result in appreciable slip. In most cases, the slip is adequately described by the Navier slip condition, which states that the slip on a boundary is proportional to the near-boundary shear stress. For Newtonian fluids, this slip condition amounts to relating the velocity slip to the velocity gradient times a slip length. The slip length $\lambda$ due to a near-wall gas "cushion" layer has been found by Vinogradova [4] to be the thickness of the gas layer $d$ multiplied by the ratio of the bulk-fluid viscosity $\mu_{c}$ to the gas viscosity $\mu_{d}$, or $\lambda=d\left(\mu_{c} / \mu_{d}\right)$.

Vinogradova's formula forms a simple yet useful basis to estimate the extent of intrinsic slip achievable on a surface. This formula, while valid in steady flow, is yet to be tested for its applicability to unsteady flow or flow in a non-inertial frame of reference. The interaction of surface slip and system rotation has been investigated by Wang [7], who applied the Navier slip condition with the same slip length to both the longitudinal and transverse flows in a rotating channel. Since the primary and secondary flows in a rotating system are interdependent, it remains to be confirmed whether the Navier slip condition in its usual form is applicable in a rotating frame of reference. This has motivated us to formulate the present problem in order to address this issue.

The present study is to look into two-fluid flow

September 8, 2016 
in a rotating environment (e.g., in a centrifuge). The two-fluid model, which assumes a homogeneous fluid core bounded by a fluid periphery of lower viscosity, has been applied by many to blood flow or flow of complex fluids. The same flow configuration as was examined by Wang [7], namely pressuredriven flow between two infinite parallel flat plates that rotate as a system about an axis perpendicular to the plates, is considered here. The specific objectives are as follows. First, based on the two-fluid model, we shall deduce the Navier slip length in the limit of a very thin depletion layer. We shall show that the slip length at its leading order is indeed equal to the one using Vinogradova's formula, irrespective of the channel rotation. At a higher order, the slip length is, however, a function of a complex rotation parameter. A complex slip length means that the velocity slip in one direction depends not only on the velocity gradient in the same direction but also on that in a normal direction. The slip length thereby becomes direction dependent. Second, we shall find out how lubrication due to a depletion layer is modulated by the channel rotation. We shall show that the primary and secondary flows may have disparate traits of dependence on the thickness of the depletion layer and the viscosity ratio. Third, we shall examine the Fåhræus-Lindqvist effect in a rotating environment. We shall show that this effect can be lessened, or even reversed, under the effect of system rotation.

\section{Problem and solution}

Figure 1 shows pressure-driven flow through a rotating parallel-plate channel of height $2 h$. The channel cross section can be divided into a core region of thickness $2(h-d)$ and a low-viscosity depletion layer of thickness $d$ near each of the two walls. Let $(u, v, w)$ be the velocity components in the Cartesian directions $(x, y, z)$, respectively, where the coordinate system rotates at a constant angular velocity $\Omega$ about the $z$-axis. We assume that the channel is so long and wide that the flow does not vary in the $x$ - and $y$-directions. It follows that $w=0$, and $u$ and $v$ are functions of $z$ only. By symmetry, it suffices for us to consider the upper half of the channel: $0 \leq z \leq h$.

The flow is primarily driven by a pressure gradient $K=-\mathrm{d} p / \mathrm{d} x$ in the axial $x$-direction, while secondary flow is induced in the transverse $y$-direction by the Coriolis force. The momentum equations

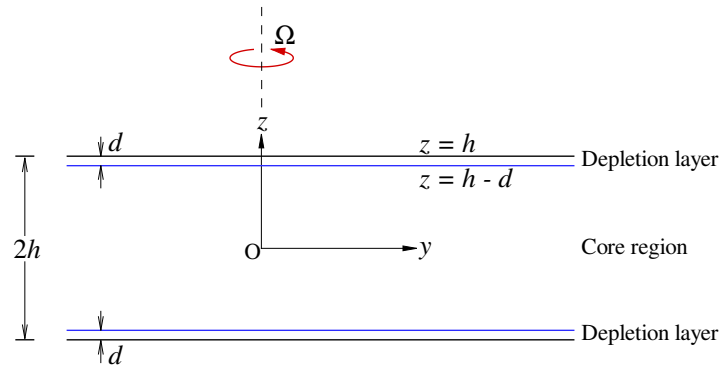

Figure 1: Definition sketch of the problem: Poiseuille flow through a rotating parallel-plate channel of height $2 h$, with a near-wall depletion layer of thickness $d$. The pressure gradient is applied in the $x$-direction, which is pointing out of the plane of the paper. The system rotates at a constant angular velocity $\Omega$ about the $z$-axis.

are as follows, where the fluid density and dynamic viscosity are denoted by $\rho$ and $\mu$, respectively.

In the core region $(0 \leq z<h-d)$ :

$$
\begin{aligned}
& -2 \rho_{c} \Omega v_{c}=K+\mu_{c} \frac{\mathrm{d}^{2} u_{c}}{\mathrm{~d} z^{2}}, \\
& 2 \rho_{c} \Omega u_{c}=\mu_{c} \frac{\mathrm{d}^{2} v_{c}}{\mathrm{~d} z^{2}} .
\end{aligned}
$$

In the depletion layer $(h-d<z \leq h)$ :

$$
\begin{aligned}
& -2 \rho_{d} \Omega v_{d}=K+\mu_{d} \frac{\mathrm{d}^{2} u_{d}}{\mathrm{~d} z^{2}}, \\
& 2 \rho_{d} \Omega u_{d}=\mu_{d} \frac{\mathrm{d}^{2} v_{d}}{\mathrm{~d} z^{2}} .
\end{aligned}
$$

We use the suffixes " $c$ " and " $d$ " to distinguish between variables of the core region and the depletion layer. The boundary and matching conditions are

$$
\begin{aligned}
& u_{c}^{\prime}=v_{c}^{\prime}=0 \quad \text { at } z=0, \\
& \left.\begin{array}{r}
u_{c}=u_{d}, v_{c}=v_{d}, \\
\mu_{c} u_{c}^{\prime}=\mu_{d} u_{d}^{\prime}, \mu_{c} v_{c}^{\prime}=\mu_{d} v_{d}^{\prime}
\end{array}\right\} \text { at } z=h-d, \\
& u_{d}=v_{d}=0 \quad \text { at } z=h .
\end{aligned}
$$

Let us introduce the following normalized variables (distinguished by carets):

$$
\left.\begin{array}{cc}
(\hat{u}, \hat{v})=(u, v) / U, & (\hat{z}, \hat{d})=(z, d) / h, \\
\hat{\Omega}_{c}=\rho_{c} h^{2} \Omega / \mu_{c}, & \hat{\Omega}_{d}=\rho_{d} h^{2} \Omega / \mu_{d},
\end{array}\right\}
$$

where the characteristic velocity scale $U \equiv K h^{2} / \mu_{c}$. In terms of these normalized variables, the momentum equations can be written in the following nondimensional form. 
For $0 \leq \hat{z}<1-\hat{d}$ :

$$
\begin{aligned}
& -2 \hat{\Omega}_{c} \hat{v}_{c}=1+\frac{\mathrm{d}^{2} \hat{u}_{c}}{\mathrm{~d} \hat{z}^{2}}, \\
& 2 \hat{\Omega}_{c} \hat{u}_{c}=\frac{\mathrm{d}^{2} \hat{v}_{c}}{\mathrm{~d} \hat{z}^{2}} .
\end{aligned}
$$

For $1-\hat{d}<\hat{z} \leq 1$ :

$$
\begin{aligned}
& -2 \hat{\Omega}_{d} \hat{v}_{d}=\kappa+\frac{\mathrm{d}^{2} \hat{u}_{d}}{\mathrm{~d} \hat{z}^{2}}, \\
& 2 \hat{\Omega}_{d} \hat{u}_{d}=\frac{\mathrm{d}^{2} \hat{v}_{d}}{\mathrm{~d} \hat{z}^{2}},
\end{aligned}
$$

where $\kappa \equiv \mu_{c} / \mu_{d}$ is the ratio of the viscosities.

Further introducing

$$
\phi_{c}=\hat{u}_{c}+\mathrm{i} \hat{v}_{c}, \quad \phi_{d}=\hat{u}_{d}+\mathrm{i} \hat{v}_{d}
$$

where $\mathrm{i}=\sqrt{-1}$ is the complex unit, the equations above can be consolidated into

$$
\begin{array}{ll}
\phi_{c}^{\prime \prime}-\sigma_{c}^{2} \phi_{c}=-1 & (0 \leq \hat{z}<1-\hat{d}), \\
\phi_{d}^{\prime \prime}-\sigma_{d}^{2} \phi_{d}=-\kappa & (1-\hat{d}<\hat{z} \leq 1),
\end{array}
$$

where

$$
\left(\sigma_{c}, \sigma_{d}\right)=(1+\mathrm{i})\left(\sqrt{\hat{\Omega}_{c}}, \sqrt{\hat{\Omega}_{d}}\right) .
$$

These functions are to satisfy $\phi_{c}^{\prime}(0)=0, \phi_{d}(1)=$ $0, \phi_{c}(1-\hat{d})=\phi_{d}(1-\hat{d})$ and $\kappa \phi_{c}^{\prime}(1-\hat{d})=$ $\phi_{d}^{\prime}(1-\hat{d})$. The solutions that satisfy these boundary/matching conditions are readily found as below.

For flow in the core region $(0 \leq \hat{z}<1-\hat{d})$,

$$
\phi_{c}(\hat{z})=c_{1} \cosh \left(\sigma_{c} \hat{z}\right)+\sigma_{c}^{-2},
$$

and for flow in the depletion layer $(1-\hat{d} \leq \hat{z} \leq 1)$,

$$
\begin{aligned}
& \phi_{d}(\hat{z})=c_{2} \sinh \left[\sigma_{d}(1-\hat{z})\right] \\
& +\kappa \sigma_{d}^{-2}\left\{1-\cosh \left[\sigma_{d}(1-\hat{z})\right]\right\},
\end{aligned}
$$

where

$$
\begin{aligned}
c_{1}= & c_{3}^{-1}\left\{-\sigma_{d} \sigma_{c}^{-2} \cosh \left(\sigma_{d} \hat{d}\right)\right. \\
& \left.+\kappa \sigma_{d}^{-1}\left[\cosh \left(\sigma_{d} \hat{d}\right)-1\right]\right\}, \\
c_{2}= & c_{3}^{-1}\left\{\kappa \sigma_{c} \sinh \left[\sigma_{c}(1-\hat{d})\right]\right. \\
& \times\left[\sigma_{c}^{-2}+\kappa \sigma_{d}^{-2}\left[\cosh \left(\sigma_{d} \hat{d}\right)-1\right]\right] \\
& \left.+\kappa \sigma_{d}^{-1} \cosh \left[\sigma_{c}(1-\hat{d})\right] \sinh \left(\sigma_{d} \hat{d}\right)\right\},
\end{aligned}
$$

and

$$
\begin{aligned}
c_{3}= & \sigma_{d} \cosh \left(\sigma_{d} \hat{d}\right) \cosh \left[\sigma_{c}(1-\hat{d})\right] \\
& +\kappa \sigma_{c} \sinh \left(\sigma_{d} \hat{d}\right) \sinh \left[\sigma_{c}(1-\hat{d})\right] .
\end{aligned}
$$

The volume flow rates in the core region and the depletion layer are then given by

$$
\begin{aligned}
Q_{c}= & Q_{c x}+\mathrm{i} Q_{c y} \\
= & 2 \int_{0}^{1-\hat{d}} \phi_{c} \mathrm{~d} \hat{z} \\
= & 2 c_{1} \sigma_{c}^{-1} \sinh \left[\sigma_{c}(1-\hat{d})\right] \\
& +2 \sigma_{c}^{-2}(1-\hat{d}), \\
Q_{d}= & Q_{d x}+\mathrm{i} Q_{d y} \\
= & 2 \int_{1-\hat{d}}^{1} \phi_{d} \mathrm{~d} \hat{z} \\
= & 2 c_{2} \sigma_{d}^{-1}\left[\cosh \left(\sigma_{d} \hat{d}\right)-1\right] \\
& +2 \kappa \sigma_{d}^{-2}\left[\hat{d}-\sigma_{d}^{-1} \sinh \left(\sigma_{d} \hat{d}\right)\right] .
\end{aligned}
$$

\section{Navier slip length as $\hat{d} \ll 1$}

We here consider the limiting case of a very thin depletion layer, the action of which on the core region thereby reduces to a Navier boundary slip condition.

\subsection{Non-rotating channel}

Let us first consider the case of a non-rotating channel: $\Omega=0$ or $\sigma_{c}=\sigma_{d}=0$, by which the solutions derived above will reduce to

$$
\left.\begin{array}{c}
\hat{u}_{c}=\frac{1}{2}\left[(1-\hat{d})^{2}-\hat{z}^{2}+\kappa\left(2 \hat{d}-\hat{d}^{2}\right)\right], \\
\hat{v}_{c}=0,
\end{array}\right\}
$$

On the interface $\hat{z}=1-\hat{d}$, the core fluid velocity and velocity gradient are

$$
\hat{u}_{c}=\kappa \hat{d}(1-\hat{d} / 2), \quad \hat{u}_{c}^{\prime}=-(1-\hat{d}),
$$

from which we may form a Navier slip condition

$$
\hat{u}_{c}=-\lambda_{0} \hat{u}_{c}^{\prime} \quad \text { on } \hat{z}=1-\hat{d},
$$

where $\lambda_{0}$ is the slip length is given by

$$
\lambda_{0}=\frac{\kappa \hat{d}(1-\hat{d} / 2)}{1-\hat{d}} .
$$


For a very thin depletion layer $\hat{d} \ll 1$, the slip length approximates to

$$
\lambda_{0} \sim \kappa \hat{d}(1+\hat{d} / 2+\cdots), \quad \hat{d} \ll 1 .
$$

At the leading order, the slip length is equal to the thickness of the depletion layer multiplied by the viscosity ratio: $\lambda_{0}^{(1)}=\kappa \hat{d}$. This accords with Vinogradova's [4] formula.

\subsection{Rotating channel}

Assuming that $\left|\sigma_{d}\right|=O(1)$ so that $\left|\sigma_{d} \hat{d}\right| \ll 1$, we may simplify the constant $c_{1}$ in Eq. (19) to

$$
c_{1} \approx \frac{\left(-1+\kappa \sigma_{c}^{2} \hat{d}^{2} / 2\right) \operatorname{sech}\left[\sigma_{c}(1-\hat{d})\right]}{\sigma_{c}^{2}\left\{1+\sigma_{c} \tanh \left[\sigma_{c}(1-\hat{d})\right] \kappa \hat{d}\right\}},
$$

where the error is $O\left(\sigma_{d} \hat{d}\right)^{3}$. Then, the velocity and velocity gradient of the core fluid on the interface $\hat{z}=1-\hat{d}$ are

$$
\begin{aligned}
\left.\phi_{c}\right|_{1-\hat{d}} \sim \frac{\kappa \hat{d}\left\{\tanh \left[\sigma_{c}(1-\hat{d})\right]+\sigma_{c} \hat{d} / 2\right\}}{\sigma_{c}\left\{1+\sigma_{c} \tanh \left[\sigma_{c}(1-\hat{d})\right] \kappa \hat{d}\right\}}, \\
\left.\phi_{c}^{\prime}\right|_{1-\hat{d}} \sim \frac{\left(-1+\kappa \sigma_{c}^{2} \hat{d}^{2} / 2\right) \tanh \left[\sigma_{c}(1-\hat{d})\right]}{\sigma_{c}\left\{1+\sigma_{c} \tanh \left[\sigma_{c}(1-\hat{d})\right] \kappa \hat{d}\right\}}
\end{aligned}
$$

from which a Navier slip condition is obtained as follows:

$$
\phi_{c}=-\lambda \phi_{c}^{\prime} \quad \text { on } \hat{z}=1-\hat{d},
$$

where the slip length

$$
\lambda \sim \kappa \hat{d}\left[1+\sigma_{c} \operatorname{coth}\left(\sigma_{c}\right) \hat{d} / 2+\cdots\right], \hat{d} \ll 1
$$

Remarkably, the slip length at the leading order is the same as that when the channel is non-rotating:

$$
\lambda^{(1)}=\lambda_{0}^{(1)}=\kappa \hat{d},
$$

which is a real quantity, implying that the velocity slip in one direction is related only to the velocity gradient in the same direction. At the next order, the slip length is

$$
\lambda^{(2)}=\sigma_{c} \operatorname{coth}\left(\sigma_{c}\right) \kappa \hat{d}^{2} / 2,
$$

which is a function of the complex rotation parameter $\sigma_{c}$. A complex slip length means that the velocity slip in one direction is correlated not only to the velocity gradient in the same direction but also to that in the normal direction. This can be manifested by writing Eqs. (33) and (34) in a matrix form:

$$
\begin{gathered}
\left(\begin{array}{l}
\hat{u}_{c} \\
\hat{v}_{c}
\end{array}\right)=-\left(\begin{array}{cc}
\lambda_{r} & -\lambda_{i} \\
\lambda_{i} & \lambda_{r}
\end{array}\right)\left(\begin{array}{l}
\hat{u}_{c}^{\prime} \\
\hat{v}_{c}^{\prime}
\end{array}\right) \\
\text { on } \hat{z}=1-\hat{d},
\end{gathered}
$$

where $\lambda_{r}$ and $\lambda_{i}$ are the real and imaginary parts of $\lambda$ given by

$$
\begin{aligned}
\lambda_{r} & =\kappa \hat{d}\{1 \\
+ & \left.\frac{\hat{d} \sqrt{\hat{\Omega}_{c}}\left[\sinh \left(2 \sqrt{\hat{\Omega}_{c}}\right)+\sin \left(2 \sqrt{\hat{\Omega}_{c}}\right)\right]}{2\left[\cosh \left(2 \sqrt{\hat{\Omega}_{c}}\right)-\cos \left(2 \sqrt{\hat{\Omega}_{c}}\right)\right]}\right\}, \\
\lambda_{i} & =\frac{\kappa \hat{d}^{2} \sqrt{\hat{\Omega}_{c}}\left[\sinh \left(2 \sqrt{\hat{\Omega}_{c}}\right)-\sin \left(2 \sqrt{\hat{\Omega}_{c}}\right)\right]}{2\left[\cosh \left(2 \sqrt{\hat{\Omega}_{c}}\right)-\cos \left(2 \sqrt{\hat{\Omega}_{c}}\right)\right]} .
\end{aligned}
$$

\section{Lubricated rotating flow}

We next look into how the core fluid flow is lubricated by the depletion layer in a rotating channel. The lubricating effect is expected to be appreciable only when $\kappa \hat{d} \geq O(1)$. Hence, for a very thin depletion layer $\hat{d} \ll 1$, the effect is significant only when the viscosity ratio $\kappa \sim \hat{d}^{-1} \gg 1$.

In Fig. 2, we show how the flow rates of the core fluid may change with the rotation parameter $\hat{\Omega}_{c}$ under the lubricating effect of a thin depletion layer $\hat{d} \ll 1$, where $\kappa=100$ and $\hat{\Omega}_{d}=\hat{\Omega}_{c}$. Figure $2(\mathrm{a})$ shows that the longitudinal flow rate $Q_{c x}$ will decrease monotonically with the rotation rate. The lubrication is to increase $Q_{c x}$ at low rotation, but is to decrease $Q_{c x}$ at high rotation. Figure 2(b) shows that the induced transverse flow rate $Q_{c y}$ will change non-monotonically with the rotation rate; the flow rate is the maximum in magnitude at an optimum rotation rate, the value of which decreases as $\hat{d}$ increases. The lubrication is always to increase the magnitude of $Q_{c y}$, where the effect is the most pronounced for $\hat{\Omega}_{c}<0.5$. The trends shown in Figs. $2(\mathrm{a}, \mathrm{b})$ are qualitatively the same as those shown in Figs. 4(a,b) of Wang [7], who studied effect of slip in rotating channel flow.

We further show in Figs. 2(c,d) the magnitude and direction of the resultant flow rate: $\left|Q_{c}\right|=$ $\left(Q_{c x}^{2}+Q_{c y}^{2}\right)^{1 / 2}$ and $\theta=\tan ^{-1}\left(Q_{c y} / Q_{c x}\right)$, as functions of $\hat{\Omega}_{c}$ and $\hat{d}$. The lubrication is always to 

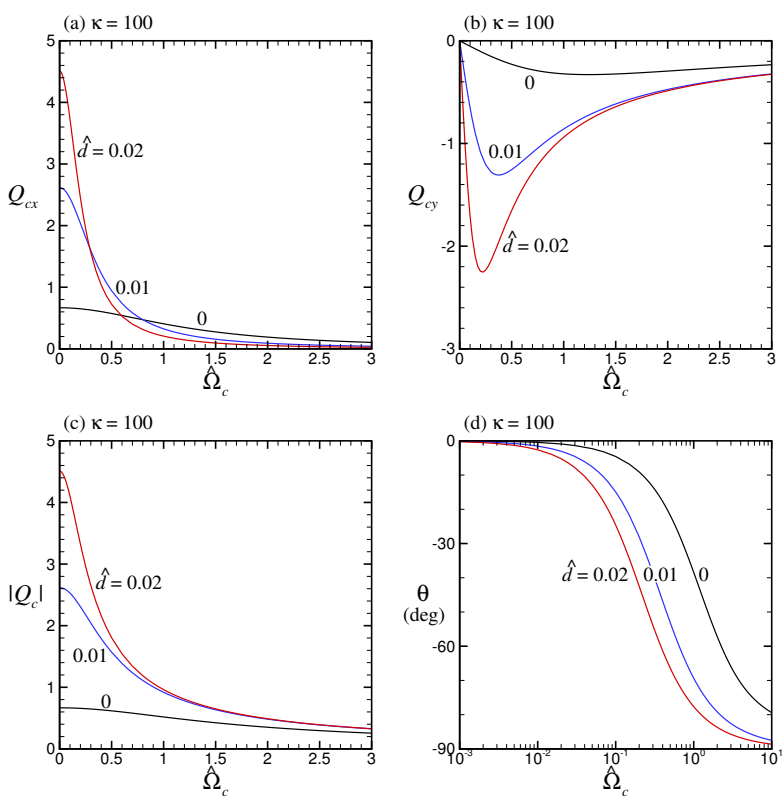

Figure 2: Flow rates: (a) $Q_{c x}$; (b) $Q_{c y}$; (c) $\left|Q_{c}\right|$; and resultant flow direction: (d) $\theta$, as functions of $\hat{\Omega}_{c}$ and $\hat{d}$, where $\kappa=100$ and $\hat{\Omega}_{d}=\hat{\Omega}_{c}$.

enhance the resultant volume transport through the channel, but the enhancing effect is appreciable only at low rotation rates. The lubrication loses much of its effect as $\hat{\Omega}_{c}$ exceeds 1 . Also, as the rotation rate increases, the resultant flow is shifted toward the negative transverse direction. Such a tendency of the flow to turn from the axial to the transverse directions is intensified under the lubrication effect.

Increasing the depletion layer thickness $\hat{d}$ leads to two opposite effects on the flow rate. One is to increase the lubricating effect, while another is to decrease the sectional area of the core region. This explains why there always exists an optimum thickness of the depletion layer $\hat{d}_{\text {optm }}$ at which the flow rate is the maximum [6]. We show in Fig. 3 how the flow rates may change with $\hat{d}$, for various values of $\kappa$, and $\hat{\Omega}_{c}=\hat{\Omega}_{d}=1$. Indeed, for any viscosity ratio $\kappa>1$, the flow rates, $Q_{c x}, Q_{c y}$ or $\left|Q_{c}\right|$, will first increase in magnitude, reaching a maximum, and then decrease in magnitude, as $\hat{d}$ increases. The optimum $\hat{d}$ for $Q_{c x}$ is smaller than that for $Q_{c y}$. Also, for $\kappa \gg 1, Q_{c x}$ may decrease very sharply from the peak when the optimum $\hat{d}$ is exceeded. We show in the inset of Fig. 3(c) the optimum depletion layer thickness $\hat{d}_{\text {optm }}$, which gives rise to the maximum $\left|Q_{c}\right|$, as a function of the viscosity ratio $\kappa$. It is seen
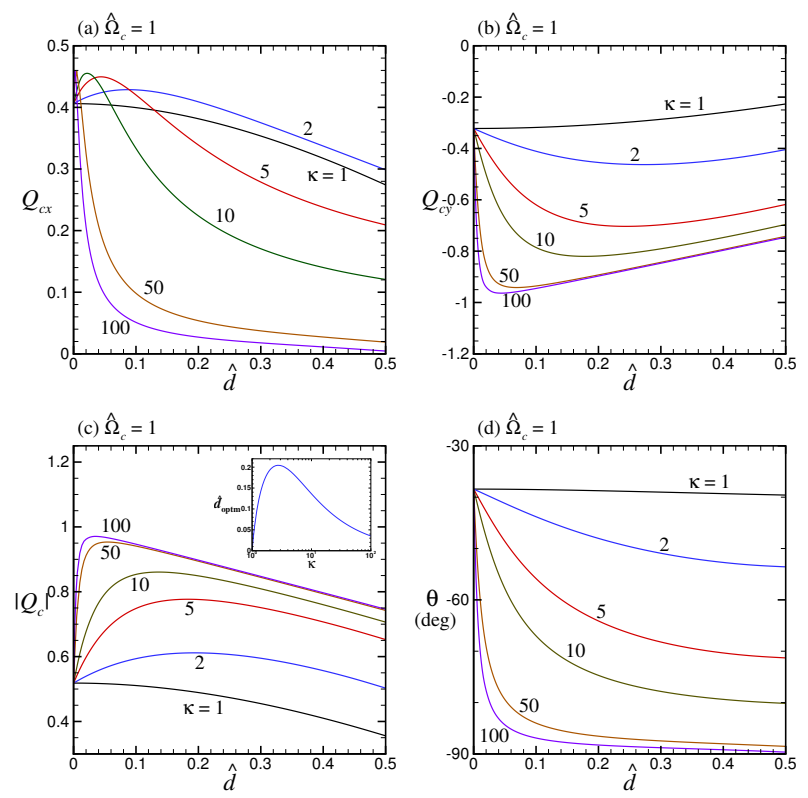

Figure 3: Flow rates: (a) $Q_{c x}$; (b) $Q_{c y}$; (c) $\left|Q_{c}\right|$; and resultant flow direction: (d) $\theta$, as functions of $\hat{d}$ and $\kappa$, where $\hat{\Omega}_{c}=\hat{\Omega}_{d}=1$. Inset of (c): optimum thickness of the depletion layer $\hat{d}_{\text {optm }}$ as a function of $\kappa$.

that $\hat{d}_{\text {optm }}$ has a peak value of approximately 0.2 at $\kappa=2.7$. For $\kappa>20, \hat{d}_{\text {optm }}$ diminishes to 0.1 or smaller. Therefore, if the viscosity ratio is high, a thin depletion layer is desirable. From Figs. 3(c,d), we see that for $\kappa=100$ the resultant flow rate $\left|Q_{c}\right|$ increases abruptly toward the peak, while the flow direction $\theta$ changes sharply toward $-90^{\circ}$, when $\hat{d}$ is slightly increased from zero.

\section{Fåhræus-Lindqvist effect in a rotating channel}

The Fåhræus-Lindqvist effect [2] refers to the phenomenon of a decreasing effective viscosity of blood traveling through a capillary tube as the tube's diameter decreases. This is essentially due to the fact that the red blood cells move mainly in the core part of the vessel, leaving a cell-free plasma layer near the wall of the vessel. The decrease of the effective viscosity was found to be most pronounced for a tube diameter less than $0.3 \mathrm{~mm}$.

Let us now examine this effect in a rotating channel by considering whole blood occupying the core region and plasma occupying the depletion layer. We shall adopt the following commonly used values (e.g., see Ethier and Simmons [8]) for the properties 
of blood: viscosity of whole blood $=3.5 \mathrm{cP}$, viscosity of plasma $=1.3 \mathrm{cP}$, density of whole blood $=$ $1060 \mathrm{~kg} / \mathrm{m}^{3}$, and density of plasma $=1025 \mathrm{~kg} / \mathrm{m}^{3}$. Based on these values, we get $\hat{\Omega}_{d}=2.6 \hat{\Omega}_{c}$ and $\kappa=2.7$.

Since flow rate in Poiseuille flow is inversely proportional to viscosity, we may define an effective viscosity, which has different components $\left(\eta_{x}, \eta_{y}\right)$ in the two directions, according to a ratio of the flow rates as follows:

$$
\eta_{x}=\frac{Q_{x}}{Q_{c x}+Q_{d x}}, \quad \eta_{y}=\frac{Q_{y}}{Q_{c y}+Q_{d y}},
$$

where

$$
Q=Q_{x}+\mathrm{i} Q_{y}=\frac{2}{\sigma_{c}^{2}}\left[1-\frac{\tanh \left(\sigma_{c}\right)}{\sigma_{c}}\right]
$$

is the flow rate if the channel were entirely occupied by the core fluid. As expected, the plasma, which is less viscous than whole blood, will act as a lubricant to the blood flow, and hence the effective viscosity is less than unity and should decrease as the fraction of the sectional area occupied by the plasma layer increases. This is the main reason for the FåhræusLindqvist effect. In a rotating channel, the same cause may not result in the same effect, however.
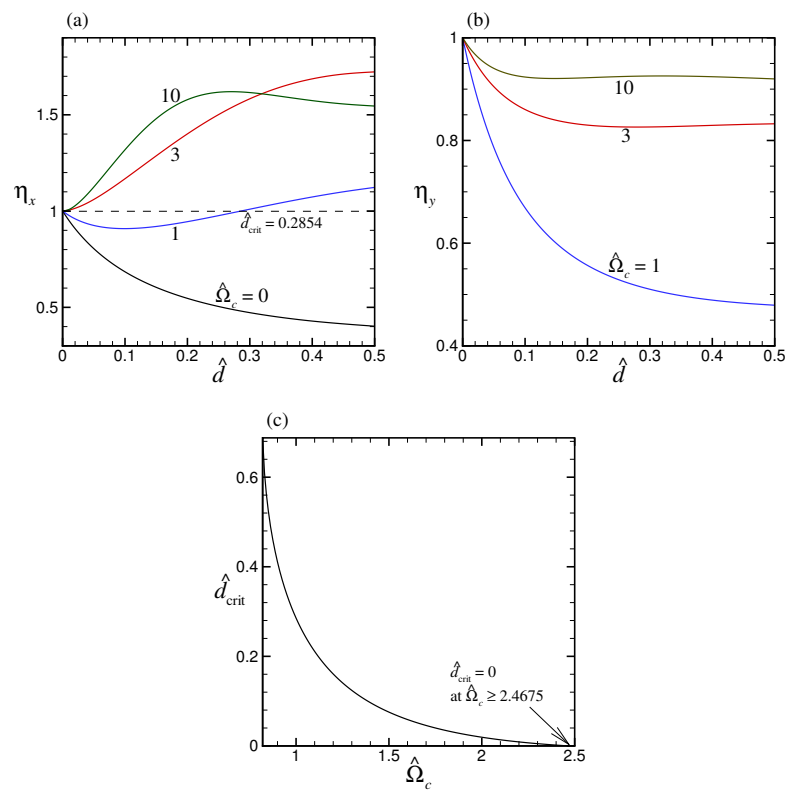

Figure 4: Effective viscosities: (a) $\eta_{x}$; (b) $\eta_{y}$, as functions of $\hat{d}$ and $\hat{\Omega}_{c}$, and (c) the critical plasma layer thickness $\hat{d}_{\text {crit }}$ as a function of $\hat{\Omega}_{c}$, where $\kappa=2.7$ and $\hat{\Omega}_{d}=2.6 \hat{\Omega}_{c}$.

We show in Figs. $4(\mathrm{a}, \mathrm{b})$ the effective viscosities $\left(\eta_{x}, \eta_{y}\right)$ as functions of $\hat{d}$ and $\hat{\Omega}_{c}$. When the channel is static $\left(\hat{\Omega}_{c}=0\right), \eta_{x}$ is always less than unity and decreases monotonically as the plasma layer thickness increases, which is consistent with the observed Fåhræus-Lindqvist effect. When the channel is rotating, the effective viscosity may exhibit disparate traits of dependence on the plasma layer thickness, depending on the rotation rate and the flow direction. For small $\hat{\Omega}_{c}$ and $\hat{d}$, the effect is still seen, in either direction. At $\hat{\Omega}_{c}=1$, $\eta_{x}$ is less than unity until the plasma layer thickness reaches a critical value $\hat{d}_{\text {crit }}=0.2854$, beyond which $\eta_{x}$ becomes larger than unity. Here, $\hat{d}_{\text {crit }}>0$ is the critical plasma layer thickness at which $\eta_{x}=1$. The Fåhræus-Lindqvist effect is observed/reversed when $\hat{d}$ is smaller/larger than $\hat{d}_{\text {crit }}$, respectively. In Fig. 4(c), the critical plasma layer thickness $\hat{d}_{\text {crit }}$ is shown as a function of $\hat{\Omega}_{c}$. We have found that $\hat{d}_{\text {crit }}$ diminishes to zero at $\hat{\Omega}_{c}=2.4675$. This means that, for $\hat{\Omega}_{c}>2.4675$ and $\hat{d}>0$, the effective viscosity in the longitudinal direction $\eta_{x}$ is always larger than unity and can get larger as $\hat{d}$ increases. The Fåhræus-Lindqvist effect will be completely reversed at high rotation rates in the direction where the pressure gradient is applied. In the transverse direction, while the effective viscosity $\eta_{y}$ remains less than unity for any $\hat{d}$ and $\hat{\Omega}_{c}$, its magnitude decreases as the rotation rate increases. We may infer from these results that the FåhræusLindqvist effect is lessened or may even be reversed in a rotating channel.

Let us gain some further insights into the problem by examining how the velocity profiles may change in character under the effect of rotation. Figures $5(\mathrm{a}, \mathrm{b})$ show that, under a low rotation rate $\hat{\Omega}_{c}=0.3$, the core fluid flow in either direction, which keeps a nearly parabolic profile, will increase in magnitude as $\hat{d}$ increases. In this case, the viscous force dominates across the core layer, and hence the lubricating effect due to the depletion layer remains influential. To the contrary, when under a higher rotation rate $\hat{\Omega}_{c}=3$, the longitudinal velocity in the core region will decrease as $\hat{d}$ increases. An explanation for this opposite effect is as follows. At high rotation, the viscous effect is limited to a thin layer, the so-called Ekman layer, near the wall. If the depletion layer is thick enough, much of the viscous effect will be confined to this layer, leaving the core region nearly inviscid. Such an inviscid core in rotating flow is known as a geostrophic core, which can be formally shown to have a uniform velocity profile (e.g., see Ped- 

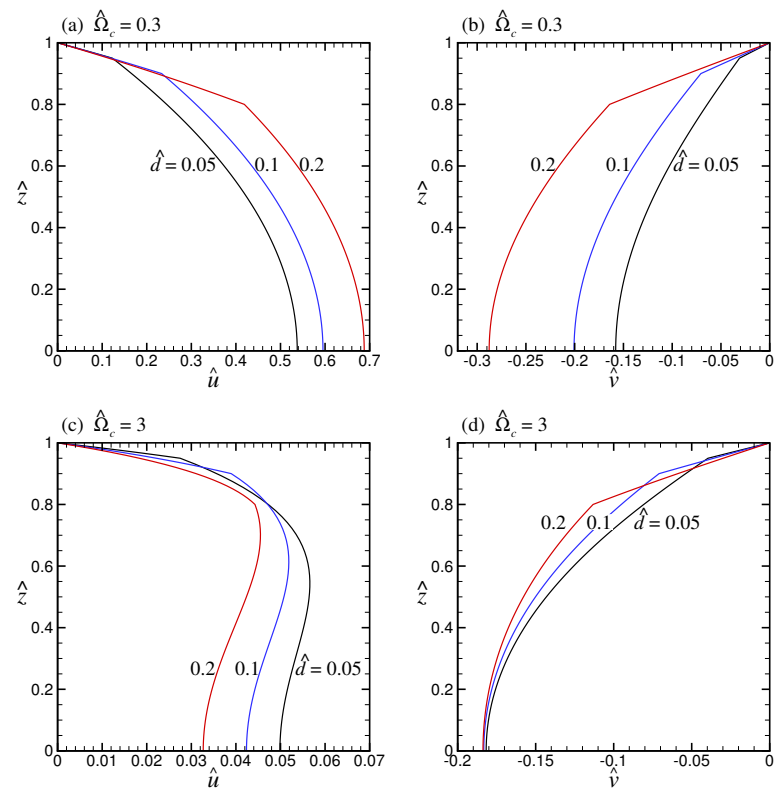

Figure 5: Velocity profiles for a low rotation rate $\hat{\Omega}_{c}=0.3$ : (a) $\hat{u}(\hat{z})$; (b) $\hat{v}(\hat{z})$, as functions of $\hat{d}$, where $\kappa=2.7$ and $\hat{\Omega}_{d}=2.6 \hat{\Omega}_{c}$. Velocity profiles for a high rotation rate $\hat{\Omega}_{c}=3$ : (c) $\hat{u}(\hat{z}) ;(\mathrm{d}) \hat{v}(\hat{z})$, as functions of $\hat{d}$, where $\kappa=2.7$ and $\hat{\Omega}_{d}=2.6 \hat{\Omega}_{c}$.

losky [9]). Such a tendency to attain a uniform core flow will inevitably result in a diminished axial velocity in the core, owing to a weakened viscous force in this region, as can be inferred from Eqs. (9) and (10). This also explains why lubrication due to a depletion layer will increasingly lose its effect as the rotational speed of the channel increases.

\section{Concluding remarks}

We have found that when the depletion layer is much thinner than the channel height, its lubricating action on fluid flow in a rotating channel is describable at the leading order by the same slip length as if the channel were non-rotating. When the depletion layer is finite in thickness, the slip length becomes a complex function of the system rotation, the thickness of the depletion layer, and the viscosity ratio. A complex slip length implies that the velocity slip depends not only on the velocity shear in the same direction but also on that in the perpendicular direction. This is a manifestation of the interdependence of the primary and secondary flows in a rotating channel. We have also found that as the rotation rate increases, the resultant flow will be more directed toward the trans- verse direction, while the lubricating effect due to the depletion layer diminishes. At a fixed rotation rate, the optimum depletion layer thickness corresponding to the maximum resultant flow rate has been found as a function of the viscosity ratio. Finally, we have explained, in terms of a geostrophic inviscid core in the limit of high rotation, why the Fåhræus-Lindqvist effect is lessened or may even be reversed in a fast rotating channel. There exists a limiting frequency above which the FåhræusLindqvist effect is always reversed in the axial direction for any $\hat{d}>0$. Below this limiting frequency, the Fåhræus-Lindqvist effect is reversed in the axial direction only when $\hat{d}$ is larger than a critical value.

\section{Acknowledgments}

Financial support was given by the Research Grants Council of the Hong Kong Special Administrative Region, China, through General Research Fund Project No. 17206615.

[1] T. Tuinier, T. Taniguchi, Polymer depletion-induced slip near an interface, J. Phys.: Condens. Matter 17 (2005) L9-L14.

[2] R. Fåhræus, T. Lindqvist, The viscosity of the blood in narrow capillary tubes, Am. J. Physiol. 96 (1931) 562-568.

[3] V. Tandon, B.J. Kirby, Zeta potential and electroosmotic mobility in microfluidic devices fabricated from hydrophobic polymers: 2. Slip and interfacial water structure, Electrophoresis 29 (2008) 1102-1114.

[4] O.I. Vinogradova, Drainage of a thin liquid film confined between hydrophobic surfaces, Langmuir 11, (1995) 2213-2220.

[5] P.G. de Gennes, On fluid/wall slippage, Langmuir 18 (2002) 3413-3414.

[6] A. Busse, N.D. Sandham, G. McHale, M.I. Newton, Change in drag, apparent slip and optimum air layer thickness for laminar flow over an idealised superhydrophobic surface, J. Fluid Mech. 717 (2013) 488-508.

[7] C.Y. Wang, The effect of slip on the flow in a rotating channel, Chem. Eng. Comm. 200 (2013) 587-594.

[8] C.R. Ethier, C.A. Simmons, Introductory Biomechanics, Cambridge University Press, Cambridge, 2007.

[9] J. Pedlosky, Geophysical Fluid Dynamics, Springer, New York, 1987. 